\title{
L'ultrafiltrazione peritoneale nello scompenso cardiaco: dall'icodestrina alla classe NYHA
}

\author{
Fulvio Floccari ${ }^{1}$, Luca Di Lullo ${ }^{2}$, Alberto Santoboni ${ }^{2}$, Moreno Malaguti ${ }^{1}$ \\ ${ }^{1}$ U.O.C. Nefrologia e Dialisi, Asl RMF, Civitavecchia (RM) \\ ${ }^{2}$ U.O.C. Nefrologia e Dialisi, Ospedale Parodi-Delfino, Colleferro (RM)
}

\begin{abstract}
Peritoneal ultrafiltration and heart failure: from icodextrin to NYHA class
Is peritoneal ultrafiltration a useful therapeutic alternative in diuretic resistant heart failure, even in patients with preserved glomerular filtration rate?

Some recent clinical trials, here briefly analysed, reported the positive effects of peritoneal ultrafiltration on NYHA class, hospitalization rates and quality of life of decompensated patients.

Why this technique has so little fortune among Nephrologists?

Keywords: Peritoneal ultrafiltration, Diuretics, Heart failure
\end{abstract}

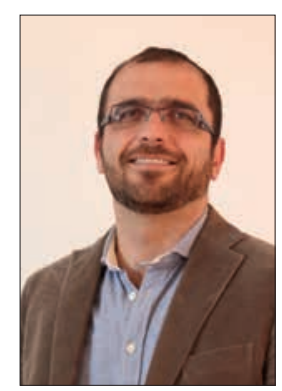

Fulvio Floccari

\section{Introduzione}

La "resistenza al diuretico" è una condizione, caratteristica delle fasi avanzate dello scompenso cardiaco, che inevitabilmente condiziona la qualità di vita del paziente, relegandolo nelle classi NYHA III (dispnea da sforzo lieve) e IV (dispnea a riposo) e costringendolo a frequenti ricoveri ospedalieri.

Anche se le moderne tecniche di "blocco sequenziale del nefrone" hanno certamente minimizzato negli anni la quota di pazienti ancora definibili come "resistenti al diuretico", per quei casi che comunque mostrano di non rispondere alla terapia medica, le tecniche di ultrafiltrazione extracorporea rappresentano un'alternativa terapeutica di diffuso utilizzo.

Non è raro osservare reparti di Cardiologia che si sono

Accepted: November 1, 2015

Published online: November 23, 2015

Indirizzo per la corrispondenza:

Dr. Fulvio Floccari

Centro di Riferimento per la Nefrologia e la Dialisi "M. Biagini"

Ospedale San Paolo

Largo dei Donatori di Sangue

00053 Civitavecchia (RM)

fulviofloccari@gmail.com dotati delle attrezzature e del know-how utili per eseguire sedute di ultrafiltrazione extracorporea, appropriandosi della metodica e affrancandosi da una Nefrologia sempre più marginale e rinunciataria.

L'analisi della letteratura scientifica documenta come l'ultrafiltrazione extracorporea garantisca al paziente minori tassi di re-ospedalizzazione (1), anche se al prezzo di un più probabile peggioramento della funzionalità renale e di un maggior numero di eventi avversi (2), oltre alla necessità di ospedalizzare i pazienti per avviarli al trattamento e di sostenerne gli elevati costi economici.

II nostro obiettivo in questa sede è quello di analizzare una terza possibilità terapeutica, trascurata dalla gran parte dei nefrologi e ancora non giunta all'attenzione dei cardiologi: l'ultrafiltrazione peritoneale (pUF).

Questa metodica di ultrafiltrazione intracorporea si propone come un'opzione di trattamento che non richiede un accesso vascolare centrale né una terapia anticoagulante, che garantisce un'ultrafiltrazione lenta e continua nel tempo e che si presta al trattamento domiciliare del paziente (3).

Piccoli studi, già dal lontano 1949, hanno riportato miglioramenti clinici e una riduzione del tasso di re-ospedalizzazione tra i pazienti affetti da resistenza ai diuretici e trattati in pUF (4-13). Malgrado ciò, la diffusione della metodica tra i pazienti affetti da scompenso è apparsa decisamente marginale.

Le dimensioni dei campioni, il disegno degli studi e la vetustà di molte pubblicazioni certamente non ne fanno un materiale ideale per analizzare il tema, ma alcune pubblicazioni più recenti hanno analizzato, in coorti di discreto calibro, i potenziali benefici della metodica (14-17). 


\section{Le recenti evidenze in letteratura}

Analizzeremo uno per uno e in maniera schematica i singoli studi, dedicando la dovuta attenzione al disegno degli stessi, alla numerosità delle coorti e alla solidità dei risultati proposti.

Considerata la rilevanza della terapia farmacologica dello scompenso ed essendosi nel tempo rese disponibili al cardiologo e al nefrologo sempre nuove opzioni terapeutiche farmacologiche (ivabradina, metolazone, ecc.), dedicheremo qualche attenzione anche alla data di apertura e chiusura di ciascun trial, sicuri che il lettore saprà trarre le proprie considerazioni su come ciascuno studio possa essere stato influenzato dalI'“ottimizzazione" della terapia medica nel corso degli anni.

Osservato, però, anche che singoli studi differiscono grandemente per la funzione renale residua dei pazienti avviati a pUF, invitiamo da subito chi ci legge a fissare la dovuta attenzione anche su questo non trascurabile aspetto di ciascun trial.

\section{Lo studio di Courivaud et al}

Il disegno dello studio. È un'analisi retrospettiva condotta ricostruendo le informazioni attraverso le cartelle cliniche ospedaliere.

Lo studio ha inizio nel 1995 e termina nel 2010.

Il campione è costituito da 126 pazienti, con un filtrato glomerulare medio decisamente conservato (in media $33 \mathrm{~mL} / \mathrm{min}$, con 69 pazienti con valori addirittura superiori a $30 \mathrm{~mL} / \mathrm{min}$ ). । pazienti con un GFR inferiore a $15 \mathrm{~mL} / \mathrm{min}$ venivano esclusi dallo studio. II trattamento, prolungato per un periodo di tempo medio di 16 mesi, era proposto, quindi, come mero trattamento ultrafiltrativo in pazienti con funzione renale sostanzialmente ancora conservata.

I risultati. Lo studio riporta un netto miglioramento della frazione di eiezione (particolarmente tra i pazienti con funzione più depressa) e una corposa riduzione del numero di giorni di ricovero per scompenso cardiaco acuto (da 3.3 giorni in media a 0.3 ), a testimonianza della gestione sostanzialmente domiciliare dei pazienti.

Gli episodi di peritonite riportati dallo studio sono complessivamente 77, corrispondenti a un episodio ogni 26.2 pazienti/mese, dei quali solo 8 tali da dover sospendere il trattamento in maniera definitiva. Cinque pazienti sono stati, in seguito, trasferiti in trattamento extracorporeo per ultrafiltrazione inadeguata.

Lo studio riporta dati completi sull'utilizzo del diuretico solo nel $48 \%$ dei pazienti, tutti in trattamento con la sola furosemide. I dosaggi medi del farmaco sono decisamente elevati, con una dose media che passa nel tempo da $560 \pm 343 \mathrm{mg}$ a $672 \pm 343 \mathrm{mg} /$ die.

I limiti. È uno studio retrospettivo di revisione delle cartelle cliniche, che manca, quindi, completamente di un gruppo di controllo che possa permettere un'analisi di mortalità. Non è previsto alcun protocollo standardizzato di terapia farma- cologica che preveda anche l'utilizzo di metolazone ed eplerenone, oltre a non fornire informazioni riguardo l'utilizzo di beta-bloccanti, ACE inibitori e altri farmaci utili nello scompenso cardiaco.

\section{L'esperienza di Koch et al}

II disegno. È uno studio prospettico, condotto su 118 pazienti incidenti con classe NYHA III o IV e insufficienza renale cronica con un filtrato medio di $19 \pm 13 \mathrm{~mL} / \mathrm{min}$ e diuresi residua di $1715 \pm 894 \mathrm{~mL} /$ die.

II trial, avviato nel 2002 e chiuso nel 2010, non ha un gruppo di controllo e tutti i pazienti arruolati sono stati, quindi, sottoposti a ultrafiltrazione intracorporea, mediante IPD trisettimanale con sedute di almeno 11 ore ciascuna.

I risultati. L'ultrafiltrazione peritoneale ha migliorato drasticamente la sintomatologia dello scompenso. Dopo 6 mesi di trattamento, sebbene siano stati osservati complessivamente ben 32 decessi, nessun paziente permaneva in classe NYHA IV (dispnea a riposo) e oltre la metà dei pazienti regrediva a uno stadio NYHA II o I.

Le dosi di furosemide utilizzate nello studio sono risultate estremamente più contenute (da $213 \pm 158 \mathrm{mg}$ a $239 \pm$ $157 \mathrm{mg} /$ die) rispetto al precedente lavoro.

L'incidenza di peritoniti riportata è di 0.53 casi per paziente/anno, quindi comparabile con quella di Courivaud et al e il trattamento è apparso sicuro e ben tollerato dai pazienti.

Non si è osservato alcun incremento della frazione di eiezione a sei mesi dall'inizio del trattamento.

I limiti. Durante il follow-up, lo studio ha subito un rapido decremento delle dimensioni della coorte (32 pazienti su 118). Non è presente alcun gruppo di controllo. La decisione di dare avvio al trattamento pUF non è presa secondo un percorso decisionale standardizzato, ma nasce da una definizione generica di scompenso refrattario.

\section{Il Registro di Heidelberg e lo studio di Frolich et al}

Il disegno. Cinquantaquattro pazienti consecutivi, affetti da scompenso cardiaco "terminale", sono stati avviati a pUF. Di questi, 39 pazienti hanno proseguito il trattamento per almeno tre mesi e, quindi, sono stati inclusi nello studio. Un gruppo di pazienti parimenti numeroso è stato, quindi, selezionato ex post dall'Heidelberg Heart Failure Registry e utilizzato come gruppo di controllo.

II GFR all'ingresso nello studio era di $22 \mathrm{~mL} / \mathrm{min}$ (intervallo di confidenza $16-30 \mathrm{~mL} / \mathrm{min}$ ) in media.

Il periodo di follow-up è breve e varia da paziente a paziente, con un range compreso tra 6 e 16 mesi.

Lo studio, pubblicato nel 2015, ha avuto luogo tra il 2006 e il 2012. Nessuna informazione viene resa circa le modalità di esecuzione della pUF.

I risultati. Lo studio conferma una riduzione dei tassi di 
ospedalizzazione per tutte le cause e per cause cardiovascolari nel gruppo trattato con ultrafiltrazione. Nessuna differenza veniva, invece, osservata in termini di mortalità da tutte le cause tra i due gruppi.

Una riduzione più significativa del peso corporeo e dei valori bioimpedenziometrici di iperidratazione veniva ottenuta nel gruppo pUF, accompagnata da un netto miglioramento della classe NYHA e della qualità di vita percepita, misurata mediante il questionario SF 36.

I pazienti sottoposti a pUf evidenziavano nel tempo un decremento significativo della diuresi (associato a un incremento del volume ultrafiltrato) e della funzione renale.

Lo studio non riporta l'incidenza di peritoniti.

I limiti. Lo studio non è randomizzato, arruola un numero decisamente piccolo di pazienti e il follow-up è breve e variabile da caso a caso. Il gruppo di controllo è stato disegnato ex post, sulla base delle caratteristiche del gruppo trattato.

\section{Un'esperienza multicentrica e italiana: Bertoli et al}

Il disegno. Studio multicentrico che ha arruolato 48 pazienti consecutivi, affetti da scompenso cardiaco "terminale" con filtrato glomerulare conservato (in media $21 \pm 10 \mathrm{~mL} / \mathrm{min}$ ).

Il periodo di follow-up è stato di 12 mesi. Lo studio, pubblicato nel 2014, ha avuto luogo tra il 2006 e il 2010.

Lo studio, nella maggioranza dei pazienti, prevedeva I'utilizzo di uno scambio unico al giorno, in modalità manuale e con il ricorso all'icodestrina, riservando l'uso del cycler in APD a una minoranza di pazienti.

I risultati. I pazienti sottoposti a pUF mostravano un'efficace riduzione del peso nel tempo, a fronte della stabilità del filtrato glomerulare. Notevoli i miglioramenti osservati nella classe NYHA, nella frazione di eiezione e nella PAPs. Netta la riduzione dei giorni di ospedalizzazione.

Lo studio non riporta l'incidenza di peritoniti.

I limiti. Lo studio non ha un gruppo di controllo, arruola un numero decisamente piccolo di pazienti e non prevede uno schema standard di trattamento in pUF, con modalità di trattamento che variano da centro a centro.

\section{Conclusioni}

Gli studi che abbiamo qui raccolto e analizzato sono estremamente eterogenei nel disegno ma accomunati tutti dell'arruolamento di pazienti che non sarebbero stati avviati alla dialisi peritoneale se non per l'esigenza di ultrafiltrarli.

Anche le modalità di esecuzione della pUF appaiono, poi, molto variegate. Pochi Autori, come Bertoli et al e Koch et al, descrivono con qualche dettaglio le modalità di esecuzione della pUF (utilizzo dell'icodestrina, schemi di trattamento secondo CAPD quotidiana o APD mediante cycler o, ancora, IPD manuale per poche sedute a settimana). La gran parte degli studi descrive molto sommariamente tali aspetti tecnici o non li descrive affatto, pur essendo questi tutt'altro che trascurabili nella lettura dei risultati.

Tuttavia, i dati che abbiamo qui riassunto consentono, a nostro giudizio, di giungere ad alcune conclusioni comunque interessanti.

Innanzitutto, appare confermato da tutti gli Autori che il ricorso alla pUF comporta un corposo miglioramento della classe NYHA e, di conseguenza, della qualità di vita del paziente, in un caso addirittura certificato attraverso il questionario SF36.

Occorre, poi, riflettere anche sul dato, ridondante nella raccolta degli studi proposta qui, di una riduzione del tasso di ospedalizzazione dei pazienti e della durata dei ricoveri.

Se la pUF, al pari dell'ultrafiltrazione extracorporea, abbia effettivamente un effetto deleterio sulla funzione renale residua o se, piuttosto, questo sia un dato in qualche misura conseguente a una più efficace disidratazione dei pazienti rimane una domanda alla quale non riteniamo sia possibile dare una risposta certa.

Il Cardionefrologo si ricordi, quindi, che dispone di un ulteriore strumento terapeutico efficace, ben tollerato e il cui costo economico appare decisamente interessante alla luce del progressivo invecchiamento della popolazione dei Paesi Occidentali. Uno strumento la cui padronanza è talmente specialistica da far correre il rischio che lo stesso rimanga inutilizzato, essendo piuttosto improbabile che i Cardiologi, approfittando ancora una volta dell'ormai proverbiale inerzia dei Nefrologi, si impossessino anche di questa tecnica.

\section{Disclosures}

Financial support: No financial support was received for this submission.

Conflict of interest: The authors have no conflict of interest.

\section{Bibliografia}

1. Bart $\mathrm{BA}$, Goldsmith $\mathrm{SR}$, Lee $\mathrm{KL}$, et al. Ultrafiltration in Decompensated Heart Failure with Cardiorenal Syndrome. N Engl J Med 2012;367:2296-304.

2. Felker GM, Mentz RJ. Diuretics and Ultrafiltration in Acute Decompensated Heart Failure. J Am Coll Cardiol 2012; 59(24):2145-53.

3. Mehrotra R, Kathuria P. Place of peritoneal dialysis in the management of treatment-resistant congestive heart failure. Kidney Int Suppl 2006;103:S67-71.

4. Schneierson SJ. Continuous peritoneal irrigation in the treatment of intractable edema of cardiac origin. Am J Med Sci 1949;218:76-9.

5. Cnossen TT, Kooman JP, Konings $\mathrm{CJ}$, et al. Peritoneal dialysis in patients with primary cardiac failure complicated by renal failure. Blood Purif 2010;30:146-52.

6. Cnossen TT, Kooman JP, Krepel HP, et al. Prospective study on clinical effects of renal replacement therapy in treatmentresistant congestive heart failure. Nephrol Dial Transplant 2012;27:2794-9.

7. Kagan A, Rapoport J. The role of peritoneal dialysis in the treatment of refractory heart failure. Nephrol Dial Transplant 
2005;20(Suppl. 7):vii28-31.

8. Nakayama M, Nakano H, Nakayama M. Novel therapeutic option for refractory heart failure in elderly patients with chronic kidney disease by incremental peritoneal dialysis. J Cardiol 2010;55:49-54.

9. Nunez J, Gonzalez M, Minana G, et al. Continuous ambulatory peritoneal dialysis as a therapeutic alternative in patients with advanced congestive heart failure. Eur J Heart Fail 2012;14:540-8.

10. Pego C, Rodrigues A, Ronco C. Role of peritoneal dialysis as a chronic renal replacement therapy in cardiorenal patients. Contrib Nephrol 2012;178:182-8.

11. Ryckelynck JP, Lobbedez T, Valette B, et al. Peritoneal ultrafiltration and treatment-resistant heart failure. Nephrol Dial Transplant 1998;13(Suppl. 4):56-9.

12. Sanchez JE, Ortega T, Rodriguez C, et al. Ef ficacy of peritoneal ultrafiltration in the treatment of refractory congestive heart failure. Nephrol Dial Transplant 2010;25:605-10.

13. Gotloib L, Fudin R, Yakubovich $M$, et al. Peritoneal dialysis in refractory end-stage congestive heart failure: a challenge facing a no-win situation. Nephrol Dial Transplant 2005;20(Suppl. 7):vii32-6.

14. Courivaud C, Kazory A, Crepin T, et al. Peritoneal dialysis reduces the number of hospitalization days in heart failure patients refractory to diuretics. Perit Dial Int 2014;34:100-8.

15. Koch $\mathrm{M}$, Haastert $\mathrm{B}$, Kohnle $\mathrm{M}$, et al. Peritoneal dialysis relieves clinical symptoms and is well tolerated in patients with refractory heart failure and chronic kidney disease. Eur J Heart Fail 2012;14:530-9.

16. Fröhlich $\mathrm{H}$, Katus $\mathrm{HA}$, Täger $\mathrm{T}$, et al. Peritoneal ultrafiltration in end-stage chronic heart failure. Clin Kidney J 2015;8:219-22.

17. Bertoli SV, Musetti C, Ciurlino D, et al. Peritoneal ultrafiltration in refractory heart failure: a cohort study. Perit Dial Int 2014;34 (1):64-70 